reactive protein in ruminants as an aid to the diagnosis of acute inflammation. Veterinary Record 88, 2-4

THORNTON, P. D. \& WATERMAN-PEARSON, A. E. (1999) Quantification of the pain and distress responses to castration in young lambs. Research in Veterinary Science 66, 107-118

WELSH, E. M., BAXTER, P. \& NOLAN, A. M. (1992) Pharmacokinetics of carprofen administered intravenously to sheep. Research in Veterinary Science $53,264-266$
WHICHER, J. T. \& WESTACOTT, C. I. (1992) The acute phase response. In Biochemistry of Inflammation. Eds J. T. Whicher, S. E. Evans. Dordrecht, Kluwer Academic Publishers. pp 243-271

WOOD, G. N., MOLONY, V., FLEETWOOD-WALKER, S. M., HODGSON, J. C. \& MELLOR, D. J. (1991) Effects of local anaesthesia and intravenous naloxone on the changes in behaviour and plasma concentrations of cortisol produced by castration and tail docking with tight rubber rings in young lambs. Research in Veterinary Science 51, 193-199

\title{
Comparison of subcutaneous ivermectin and oral moxidectin for the treatment of notoedric acariasis in hamsters
}

\author{
L. Beco, A. Petite, T. Olivry
}

\begin{abstract}
Thirty hamsters diagnosed with a Notoedres infestation on the basis of their clinical signs and skin scrapings were allocated to three matched groups. The hamsters in group 1 received ivermectin at $400 \mu \mathrm{g} / \mathrm{kg}$ subcutaneously once a week for eight weeks, those in group 2 were treated with moxidectin at $400 \mu \mathrm{g} / \mathrm{kg}$ orally once a week, and those in group 3 were treated with moxidectin at the same dosage, but twice a week. The hamsters' skin lesions were scored weekly on the basis of the severity of crusting, erythema, scaling and excoriations at various sites. In all three groups the lesion scores were significantly lower after four and eight weeks, and there was no significant difference between the efficacy of the treatments. However, at the end of the treatment, skin scrapings were negative in only 60 to 70 per cent of the animals in each group.
\end{abstract}

NOTOEDRIC acariasis is a parasitic infestation which is rarely reported in hamsters (Baies and others 1968) and there are no clear data on its incidence and prevalence. The clinical signs consist of pruritus, erythema, crusts, erosions and hyperpigmentation. The skin lesions are usually observed on the ear pinnae, face, genitalia, tail and limbs, but they can be distributed more generally. The infestation is very contagious and can affect an entire colony when the hamsters are kept together. As with other sarcoptid mite infestations natural infestations are presumed to be due to direct contact with affected animals. There are no reports of treatment protocols for Notoedres infestation in hamsters, but because avermectin drugs are routinely used for the treatment of this parasitic infestation in small mammalian species (Smith and Burgmann 1997, Adamcak and Otten 2000), it is assumed that they should be effective in hamsters.

This study was designed to compare three therapeutic protocols for treating notoedric acariasis in hamsters. The efficacy, side effects and ease of use of weekly injections of ivermectin were compared with weekly or twice weekly oral doses of moxidectin in a colony of 30 hamsters treated for eight weeks.

\section{MATERIALS AND METHODS}

\section{Study design and sample size}

The experiment was planned as a prospective, open, parallel trial without crossover. Ten hamsters per group were used to give a 95 per cent power to detect a 65 per cent difference in clinical scores ( $s d=33$ per cent; $P=0.01$ ) between the three treatment groups.

\section{Subjects and inclusion criteria}

Thirty hamsters were selected at the same time from a pet shop; apart from skin lesions they appeared healthy. All animals exhibited severe pruritus and cutaneous lesions consisting of erythema, crusting, excoriations and hyperpigmentation (Fig 1). The lesions were predominantly on the face, ears, medial thighs, genitalia and tail. Skin scrapings contained numerous mites identified as Notoedres species (Fig 2). The hamsters were allocated to three equal groups matched with respect to sex ratio, weight, type of coat and severity of clinical signs. The three groups of hamsters were maintained in similar environmental conditions and were fed the same diet.

\section{Treatment groups}

The first group of 10 hamsters was treated with subcutaneous injections of $400 \mu \mathrm{g} / \mathrm{kg}$ ivermectin (Ivomec; MSD-AGVET) once a week for eight weeks. The second group of 10 hamsters received $400 \mu \mathrm{g} / \mathrm{kg}$ moxidectin (Cydectin ovin $0 \cdot 1$ per cent oral solution; Fort Dodge Animal Health) orally once a week for eight weeks, and the third group of 10 hamsters was given moxidectin orally at the same dosage, but twice a week for eight weeks. The second dosage of moxidectin was selected because it had been shown to be efficacious for the treatment of canine demodicosis in a pilot trial (Bensignor and Carlotti 1998). These protocols constitute an extralabel use of drugs not approved for administration in hamsters.

\section{Assessment of response to therapy}

Evaluation of skin lesions Before and at each weekly re-evaluation the hamsters were given a clinical score by assessing the severity (mild $=1$; moderate $=2$; severe $=3$ ) of four skin lesions (erythema, crusts, excoriations and lichenification)
Veterinary Record (2001) 149, 324-327

L. Beco, DrMedVet, A. Petite, DrMedVet, Clinique Veterinaire Avenue Reine Astrid 104, B-4900, Spa, Belgium T. Olivry, DrVet, $\mathrm{PhD}$, Department of Clinical Sciences, College of Veterinary Medicine, North Carolina State University, Raleigh, North Carolina, USA 
FIG 1: Hamster with lesions of Notoedres infestation on its face and feet. Severe crusting can be observed on the muzzle ears and extremities

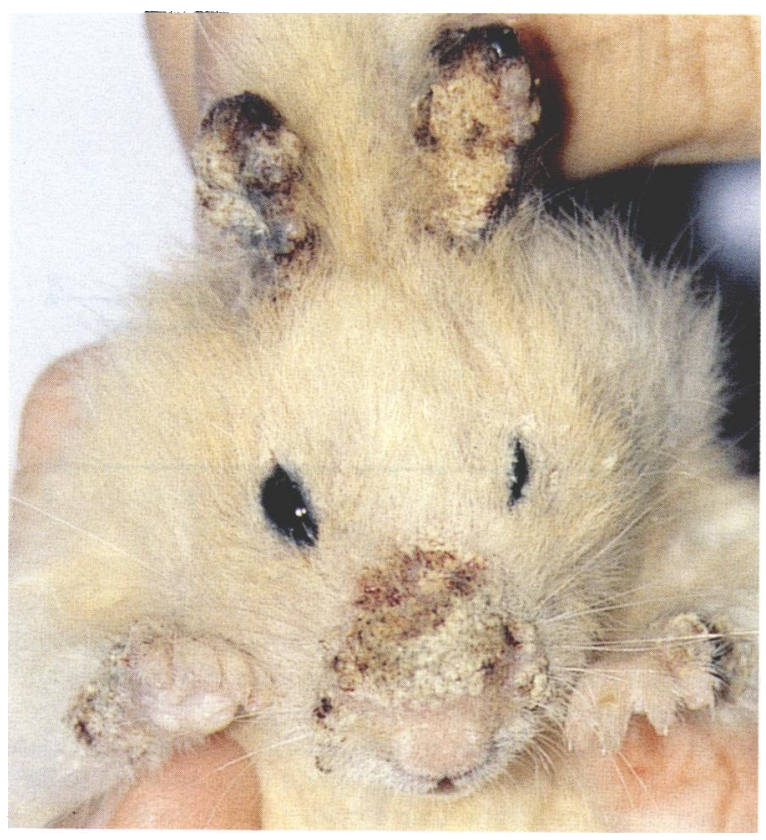

at five body sites (face, ears, tail, genitalia and inner thighs). The maximum possible score was $3 \times 4 \times 5=60$.

Evaluation of pruritus Before the treatments began and each week for eight weeks, a pruritus score was calculated for each group of hamsters. It consisted of the number of episodes of pruritic activity observed in each group during a period of 30 minutes, divided by the number of hamsters in the group.

Evaluation of mite infestation Before and at each reevaluation visit, one skin scraping was taken from the ear pinnae of each hamster. Each scraping was collected in chloral lactophenol and examined under $100 \times$ magnification. Notoedric mites, their progeny, or their faecal pellets were recorded as either present or absent after the examination of $1.8 \mathrm{~cm}^{2}$ of slide surface. The results were expressed as the percentage of hamsters with positive scrapings in each group.

\section{Statistical analyses}

Within each group, the weekly clinical scores were compared by means of a non-parametric repeated-measures analysis of variance (Friedman test) by using statistical software (Prism; GraphPad). The clinical scores of the three groups were compared by means of a non-parametric Kruskal-Wallis analysis of variance.

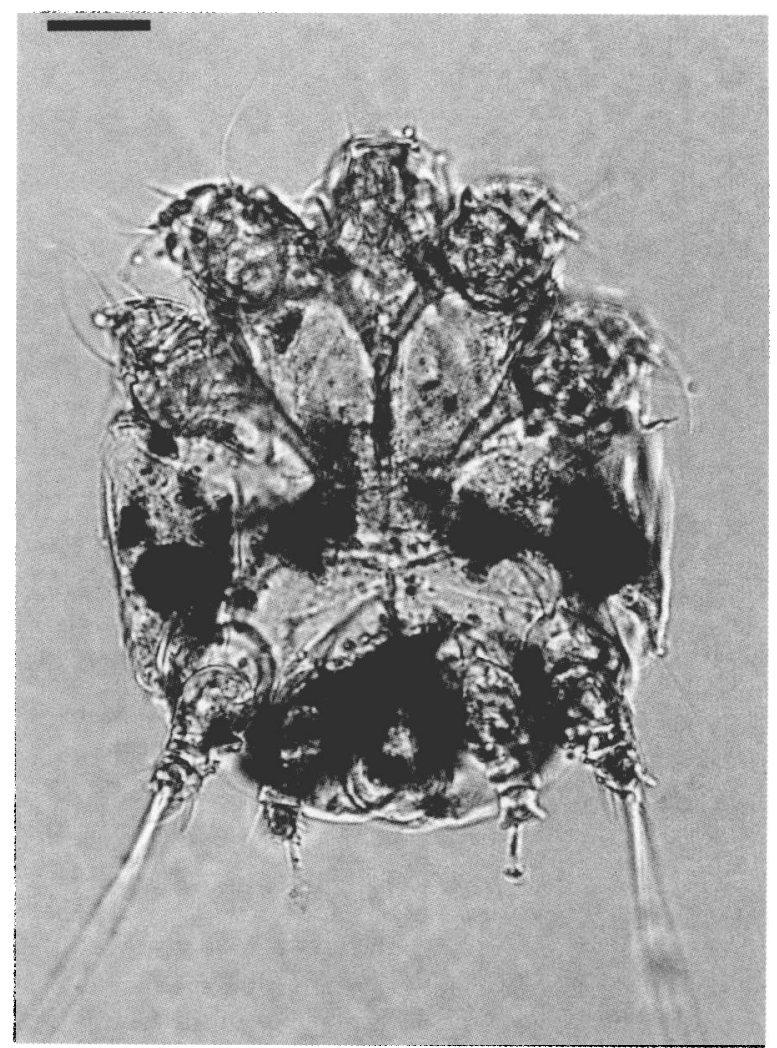

FIG 2: Notoedres mite, characterised by a dorsal anus. Skin scraping; chloral lactophenol. Scale bar $=25 \mu \mathrm{m}$

\section{RESULTS}

\section{Clinical scores}

After four weeks of treatment the clinical scores of the groups were not significantly different from before the beginning of the trial. However, after eight weeks, there was a significant reduction $(\mathrm{P}<0.0001$, Fig 3$)$. At the end of the study, the median percentage reductions in the clinical scores were 89 per cent in group 1,86 per cent in group 2 , and 95 per cent in group 3 . There were no significant differences between the clinical scores of the three treatment groups after either four or eight weeks.

\section{Pruritus assessment}

The pruritus scores decreased progressively during the trial in the three groups of hamsters (Fig 4), and after four and eight weeks of treatment there were no differences between the scores of the three groups.
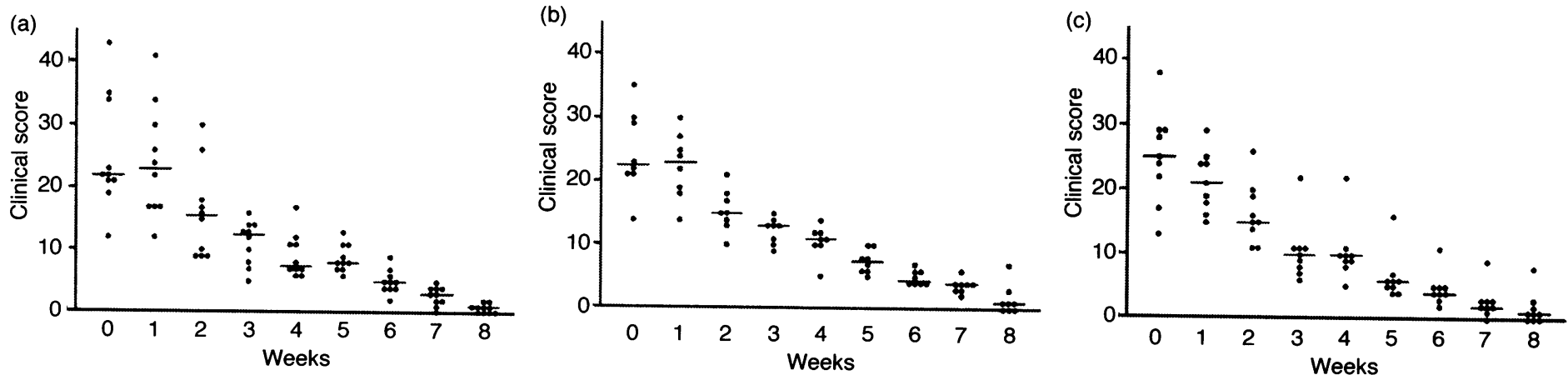

FIG 3: Clinical scores in the three groups of hamsters during treatment with (a) ivermectin subcutaneously once a week, (b) moxidectin orally once a week, and (c) moxidectin orally twice a week. Each dot represents one hamster. At each time point the bar indicates the median score 


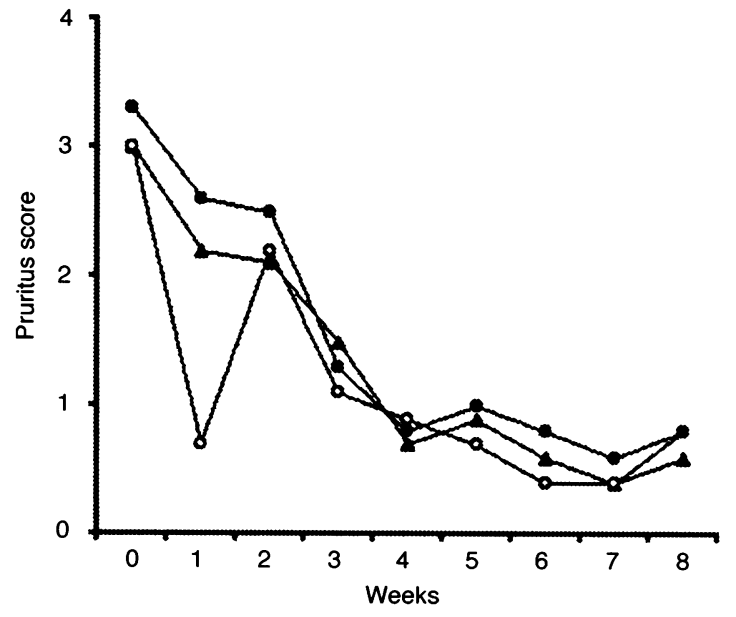

FIG 4: Pruritus scores in the three groups of hamsters during treatment with ivermectin or moxidectin. Each symbol represents the mean pruritus score of a group of hamsters. $\Delta-\Delta$ Group 1, ivermectin subcutaneously once a week: $\bigcirc-\bigcirc$ Group 2, moxidectin orally once a week; -0 Group 3, moxidectin orally twice a week

\section{Mite infestation}

During the first three weeks of therapy, the skin scrapings from all the hamsters yielded numerous mites or faecal pellets, but subsequently the percentage of positive scrapings decreased similarly in the three treatment groups (Fig 5). At the end of the study, the skin scrapings remained positive in four of the 10 hamsters in group 1 , in two of the eight remaining hamsters in group 2, and in four of the nine remaining hamsters in group 3.

\section{Treatment side effects}

In group 1 , the subcutaneous injections of ivermectin induced signs of pain and led to difficulty in handling the hamsters. No side effects were observed in the hamsters receiving moxidectin. During the trial, two of the hamsters in group 2 and one in group 3 died from unknown causes, one of those in group 2 was cannibalised. A multicentric lymphosarcoma was diagnosed in both the other hamsters which died, but no causal relationship between moxidectin and the lymphosarcoma could be established.

\section{DISCUSSION}

The results of this trial provide evidence that oral moxidectin is as effective as subcutaneous ivermectin for the reduction of the clinical signs and parasitic load in hamsters infested with Notoedres species. However, the eight-week period of treatment with either drug was not long enough to obtain clinical remission and eliminate the mites completely.

A significant clinical improvement was observed in the hamsters treated with either ivermectin or moxidectin only after three weeks. Even after eight weeks of therapy some skin lesions persisted, especially on the ear pinnae and genitalia. The hamsters most severely affected at the beginning of the trial appeared to be the most difficult to cure. This length of therapy and partial cure rate are notably different from the results reported for the ivermectin therapy of Notoedres infestation in other mammalian species (Evans 1984, Klompen and Nachman 1990, Maehr and others 1995, Anon 2000). The lower efficacy observed in this trial may have been due first to the severity of the infestation at the beginning of the treatment; secondly, the fact that the hamsters were housed together in the same restricted environment may have

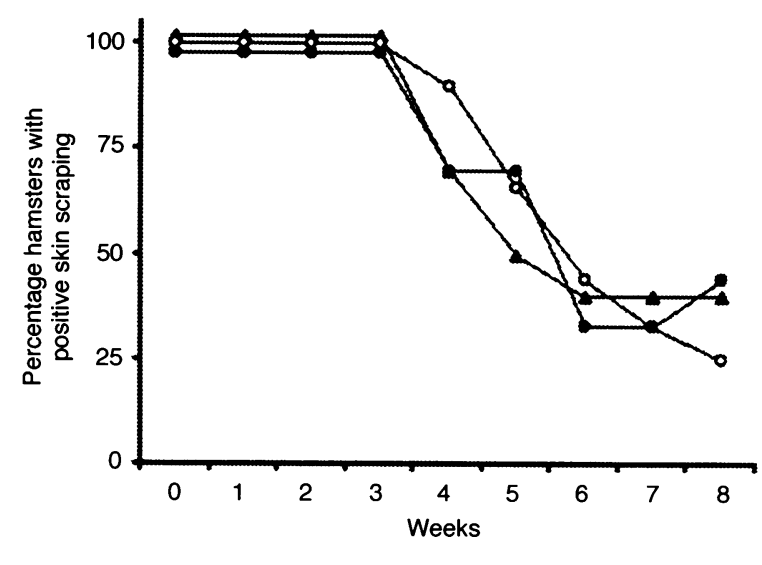

FIG 5: Evaluation of mite infestation in the three groups of hamsters during treatment with ivermectin or moxidectin. Each symbol represents the percentage of hamsters in a group with positive skin scrapings. $\Delta-\Delta$ Group 1, ivermectin subcutaneously once a week; $\bigcirc-\bigcirc$ Group 2, moxidectin orally once a week; -O Group 3, moxidectin orally twice a week induced a stress response leading to immunosuppression and permanent reinfestation. Thirdly, the doses of ivermectin and moxidectin used had been extrapolated from the doses proposed for the treatment of acariasis in other mammalian species. It is possible that either the dosage and/or the frequency of administration were inadequate to treat an infestation with Notoedres in hamsters.

The hamsters treated with ivermectin showed signs of pain when they were injected subcutaneously, and they reacted vigorously and showed a propensity to bite. Signs of skin necrosis have also been observed occasionally at the site of ivermectin injections in other hamsters with acariasis (L. Beco, unpublished observations). In contrast, moxidectin was easier to administer, with the oral solution being readily accepted by the animals. The oral route also makes it possible for the owners to treat their pets at home. No obvious side effects were observed in the hamsters receiving moxidectin, although it is uncertain whether the development of lymphosarcoma in two of the hamsters was related to the drug. Moxidectin has been shown to be non-mutagenic and noncarcinogenic in safety studies in several species of rodent (European Agency for Evaluation of Medicinal Products 1997).

Future studies should evaluate whether oral moxidectin would be more effective if administered more frequently or for longer periods. Alternatively, the use of other avermectins could be considered. Doramectin is a new injectable avermectin with a half-life twice that of ivermectin. Recently, a single injection of doramectin effectively cured notoedric acariasis in five cats in a cat shelter, and there were no signs of pain when they were injected and no adverse reactions were observed (Delucchi and Castro 2000). The novel avermectin selamectin is effective against Sarcoptes scabiei when applied topically (Shanks and others 2000) and it might also be helpful for treating Notoedres infestations in various mammalian species.

\section{ACKNOWLEDGEMENTS}

The authors thank Professor Patrick Bourdeau for his help in the identification of the mite, and Dr Marianne Heiman for postmortem and histopathological analyses of the hamsters which died.

\section{References}

ANON (2000) Wratachtige woekeringen op de oren van ratten. Tijdschrift voor Diergeneeskunde 125, 337-338

ADAMCAK, A. \& OTTEN, B. (2000) Rodent therapeutics. Veterinary Clinics of North America: Exotic Animal Practice 3, 221-237

BAIES, E., SUTEU, I. \& KLEMM, W. (1968) Notoedres scabies in the golden hamster. Zeitung Versuchstierkunde 10, 251-257 
BENSIGNOR, E. \& CARLOTTI, D. N. (1998) Moxidectin in the treatment of generalised demodicosis in dogs: a pilot study: 8 cases. In Advances in Veterinary Dermatology. Vol 3. Eds K. W. Kurochka, T. Willemse, C. von Tscharner. Oxford, Butterworth-Heinemann. pp 554-555

DELUCCHI, L. \& CASTRO, E. (2000) Use of doramectin for treatment of notoedric mange in five cats. Journal of the American Veterinary Medical Association 216, 215-216

EUROPEAN AGENCY FOR EVALUATION OF MEDICINAL PRODUCTS (1997) Committee for Veterinary Medicinal Products. Moxidectin. http:// www.emea.eu.int/pdfs/vet/mrls/Moxidectinl.pdf. Accessed June 19, 2001

EVANS, R. H. (1984) Ivermectin treatment of notoedric mange in two fox squirrels. Journal of the American Veterinary Medical Association 185, 14371438
KLOMPEN, J. S. H. \& NACHMAN, M. W. (1990) Occurrence and treatment of the mange mite Notoedres muris in Marsh rats from South America. Journal of Wildlife Diseases 26, 135-136

MAEHR, D. S., GREINER, E. C., LANIER, J. E. \& MURPHY, D. (1995) Notoedric mange in the Florida panther (Felis concolour coryi). Journal of Wildlife Diseases 31, 251-254

SHANKS, D. J., MCTIER, T L., BEHAN, S, PENGO, G., GENCHI, C., BOWMAN, D. D., HOLBERT, M. S., SMITH, D. G., JERNIGAN, A. D. \& ROWAN, T. G. (2000) The efficacy of selamectin in the treatment of naturally acquired infestations of Sarcoptes scabiei on dogs. Veterinary Parasitology 91, 269-281 SMITH, D. A. \& BURGMANN, P. M. (1997) Formulary. In Ferrets, Rabbits and Rodents. Clinical Medicine and Surgery. Eds E. V. Hillyer, K. F. Quesenberry. Philadelphia, W. B. Saunders. pp 392-403

\section{Short Communications}

\section{Diffuse splenomegaly caused by splenic abscessation in a dog}

\author{
P. J. Ginel, R. LUCENA, J. AROLA, \\ M. P. MARTIN, E. MOZOS
}

SPLENIC abscesses represent a rare cause of chronic inflammation of the spleen, most usually manifested by a localised splenomegaly (Neer 1996). In dogs, localised splenomegaly is more common than diffuse or generalised splenomegaly, and other reported aetiologies include haematoma formation, nodular hyperplasia, infarction, neoplasia and cyst formation after trauma-induced haematoma (Neer 1996, Couto and Gamblin 2000). On the other hand, diffuse splenomegaly has been associated with different types of splenitis, lymphoreticular hyperplasia, congestion and infiltration by neoplastic cells (Neer 1996).

Most clinical reports that specifically consider the canine spleen, focus on different clinical aspects of splenic neoplasia, mainly haemangiosarcoma and infectious causes (Spangler and Culbertson 1992, Valli and Parry 1993, Day and others 1995), whereas splenic abscesses are infrequently cited and always as a cause of localised disease. This case report describes the clinical and pathological aspects of diffuse splenomegaly caused by complete splenic abscessation in a dog. To the authors' knowledge, this condition has not been reported previously in the dog.

A five-year-old intact male Spanish mastiff was referred to the authors' clinic because of progressive chronic weight loss and intermittent diarrhoea of eight weeks duration. The dog was regularly vaccinated and lived outdoors as a guard dog. At first examination, the dog was emaciated with severe muscle atrophy. Its mucous membranes were pale and its rectal temperature was $41^{\circ} \mathrm{C}$. Palpation of the abdomen was non-painful and apart from a mild, diffuse splenomegaly it was considered normal. A fine-needle aspiration biopsy of the popliteal lymph node showed numerous Leishmania amastigotes. No other abnormalities could be found and all clinical signs were attributed to Leishmania infection. Treatment consisted of 20 $\mathrm{mg} / \mathrm{kg} /$ day of allopurinol (Zyloric 300; Laboratorios FAES). The dog was lost to follow-up but three months later it returned to the clinic with severe cachexia, hindlimb weakness and a pen- dulous abdomen. Abdominal palpation was painful and revealed a diffuse splenomegaly. A haemogram showed a low haematocrit value ( 23 per cent), severe neutrophilic leucocytosis $\left(43.8 \times 10^{3}\right.$ leucocytes $\left./ \mu \mathrm{l}\right)$ and anaemia $\left(2.98 \times 10^{6}\right.$ erythrocytes $/ \mu \mathrm{l})$, thrombocytopenia $(176,000$ platelets/ $\mu \mathrm{l})$, low haemoglobin levels $(7.5 \mathrm{~g} / \mathrm{dl})$ and elevated serum total protein $(10.2 \mathrm{~g} / \mathrm{dl})$. Altered serum biochemical parameters included high serum alkaline phosphatase activity (956 iu/litre). Bilirubinuria and low urine specific gravity $(1010)$ were detected after the urinalysis. Radiographic examination showed a large radiopaque mass located caudoventrally to the liver with poorly defined margins suggestive of splenomegaly and peritonitis. Abdominal ultrasonography revealed an enlarged and ill-defined spleen with a predominantly hypoechoic to anechoic appearance and moderate deep acoustic enhancement (Fig 1). Several small hyperechoic foci with minimal acoustic shadowing, as seen in gas-containing lesions, could be identified but were not prominent (Ralls and others 1982). Although abscesses are usually easily distinguishable from the surrounding homogeneous normal spleen (Ralls and others 1982), in this case the lesion seemed to involve the whole organ, and areas with a normal sonographic granular splenic pattern were not found. The owner declined any further diagnostic testing and the dog was euthanased.

At necropsy, all abdominal seroses were covered by an abundant and thick fibrinopurulent exudate. Abdominal organs showed evidence of autolysis and putrefaction although the necropsy was performed four hours after euthanasia and the cadaver had been refrigerated. The liver was pale, friable and foamy because of putrefaction gases. The spleen was enlarged and had lost its normal morphology showing an oval shape with a surface covered by the same fibrinopurulent exudate ( $\mathrm{Fig} 2$ ). On palpation, the organ was fluctuant as if filled with abundant fluid and, when sectioned, a large amount of yellow-greenish liquid exudate was obtained (Fig 2). The large abscess was well capsulated and only a small proportion of recognisable splenic parenchyma was present at one pole of the organ. Both kidneys appeared diffusely pale and friable. A macroscopic diagnosis of massive splenic abscessation and severe secondary fibrinopurulent peritonitis was made.

Microscopically, the peritoneal surface and organ capsules were diffusely infiltrated by fibrin strands, mixed inflammatory cells and granulation tissue of variable maturity. The infiltrate was composed predominantly of neutrophils, macrophages, plasma cells and mature lymphocytes. The wall of the abscess was composed of a fibrous stroma and mature 